\title{
Censo de las Unidades del Dolor en España. Análisis de la estructura organizativa, dotación, cartera de servicios e indicadores de calidad y buenas prácticas
}

\author{
J. R. González-Escalada ${ }^{1}$, A. Camba² e I. Sánchez ${ }^{3}$ \\ ${ }^{1}$ Unidad del Dolor. Hospital Universitario Ramón y Cajal. Madrid. ${ }^{2}$ Unidad del Dolor. Hospital Arquitecto \\ Marcide. Ferrol, La Coruña. ${ }^{3}$ Dirección Médica. Grunenthal. España
}

González-Escalada JR, Camba A, Sánchez I. Censo de las Unidades del Dolor en España. Análisis de la estructura organizativa, dotación, cartera de servicios e indicadores de calidad y buenas prácticas. Rev Soc Esp Dolor 2014; 21(3): 149-161.

\begin{abstract}
According to the census in the existing Spanish Society of Pain (SED) in February 2012 were recorded 147 Units Pain in Spain. Thanks to the collaboration of the Foundation Signo (Foundation which represents health managers), after comparing their files, with directions of hospitals public and institutions non-public registered by the Ministry of Health, 36 units were added to book entries in the SED, which has identified a total of 183 units in Spain. All of them were asked to collaborate with an online survey hosted on a particular web, order to know the crew and the degree of complexity of each, as well as compliance with the Standards Quality and Best Practices of Pain Units, edited by the Ministry of Health in 2010. One hundred and forty-three responses were considered valid surveys which accounted for $80.3 \%$ of all units surveyed, and thus a very representative sample. In this paper are analyzed the results obtained in terms of the structure, endowment portfolio of services and activities of each and contrasted with the definition of the Units issued by the $\mathrm{Mi}$ nistry. We conclude that it is necessary to reconsider this, and propose a classification according to the actual situation Units that would reflect our strengths and weaknesses and serve as a reference and starting point for improving pain care in our country. To validate the results of an audit conducted by random selection of eight units (5.7\% of respondents), with a varying degree of overlap between $72-100 \%$ in the diffe-
\end{abstract}

Recibido: 01-06-13.

Aceptado: 01-09-13. rent sections and a degree of overall match exceeding $86 \%$, which is the average value of truthfulness we consider for the first Census of pain units in Spain, with a confidence interval ranging between 81 and $90 \%$.

Key words: Pain Units. Number. Structure. Staffing. Quality standard. Census in Spain.

\section{RESUMEN}

Según el censo existente en la Sociedad Española del Dolor (SED) en febrero de 2012 se contabilizaban 147 Unidades del Dolor. Gracias a la colaboración de la Fundación Signo (fundación que agrupa a los gestores de sanidad), tras cotejar sus ficheros con las direcciones-gerencias de centros hospitalarios públicos y las registradas por el Ministerio de Sanidad en entidades no públicas, se sumaron 36 Unidades a las censadas en la SED, lo que ha permitido identificar un total de 183 Unidades en España. A todas ellas se les solicitó colaborar con una encuesta online hospedada en una hoja web específica, con el objetivo de conocer la dotación y el grado de complejidad de cada una de ellas, así como el cumplimiento de los Estándares de Calidad y Buenas Prácticas de las Unidades del Dolor, editado por el Ministerio de Sanidad en el año 2010. Después de analizar las respuestas se consideraron válidas 143 encuestas, lo que suponía el 80,3\% del total de las Unidades censadas, y por tanto una muestra muy representativa. En este trabajo se analizan los resultados obtenidos en cuanto a la estructura, dotación, cartera de servicios y actividad de cada una de ellas y se contrasta con la tipificación de las Unidades dictada por el Ministerio. Se concluye que es necesario reconsiderar esta clasificación, proponiendo una más acorde con la situación real de las Unidades, que sea fiel reflejo de nuestras fortalezas y debilidades y sirva de referencia y punto de partida para mejorar la atención al dolor en nuestro país. Para validar los resultados obtenidos, una vez recogidas todas las encuestas se realizó una auditoría mediante selección aleatoria de ocho 
Rev. Soc. Esp. del Dolor, Vol. 21, N. ${ }^{\circ}$ 3, Mayo-Junio 2014

Unidades (5,7 \% de las encuestadas), con un grado de coincidencia que oscila entre el 72-100\% en los distintos apartados y un grado de coincidencia global que supera el $86 \%$, que es el valor medio de veracidad que consideramos para este primer Censo de la Unidades del Dolor en España, con un intervalo de confianza que se sitúa entre el 81 y el $90 \%$.

Palabras clave: Unidades del Dolor. Número. Estructura. Dotación. Estándar de calidad. Censo en España.

\section{INTRODUCCIÓN}

El dolor crónico es uno de los problemas de salud que más preocupa a los españoles. Cerca de cinco millones y medio de españoles (más del $25 \%$ de los adultos mayores de 15 años) padecen dolor crónico. En todos los países, el dolor crónico es más frecuente en las mujeres y su incidencia aumenta con la edad $(1,2)$ por lo que el problema se agravará con el paso del tiempo debido al envejecimiento de la población. Aunque la prevalencia del dolor crónico en la población es muy alta, todos los involucrados (usuarios y profesionales) identifican múltiples carencias en su tratamiento $(3,4)$. Muchos pacientes pasan de un especialista a otro sin obtener una solución aceptable para su problema y la satisfacción con la atención que reciben es muy deficiente y variable, con grandes diferencias entre las distintas autonomías $(5,6)$. El problema se agrava en el medio rural, donde el acceso a los centros especializados es necesariamente más complicado, por lo que las deficiencias se magnifican en aquellas regiones y autonomías con menor densidad poblacional y por tanto, mayor dispersión de los recursos sanitarios. Según la encuesta "Pain in Europe", realizada en 15 países europeos e Israel (7), el $40 \%$ de los pacientes con dolor crónico opinaban que no estaban recibiendo el tratamiento adecuado para su dolor, a pesar de que el $34 \%$ lo describieron como dolor severo. Como media, los pacientes tardaron unos 3,6 años en recibir tratamiento especializado en una Unidad del Dolor, y todos ellos habían pasado por múltiples especialidades antes de ser derivados a la Unidad.

El dolor crónico sigue siendo un problema de salud pública sin resolver, con un coste económico muy elevado (8). Desde todos los ámbitos se reconoce la necesidad de dotar al sistema sanitario de equipos especializados y multidisciplinarios, con una formación integral en el tratamiento del dolor crónico $(9,10)$. El interés de las competencias en dolor se deduce de los objetivos de los planes de salud, que obligan a las instituciones sanitarias competentes a tener organizada esta asistencia mediante Unidades de Tratamiento del Dolor Crónico en el ámbito hospitalario, con una cartera de servicios claramente definida (11). Para la correcta disposición de los recursos en este campo sería necesario identificar las carencias del sistema a partir del conocimiento y reconocimiento de la situación real y actualizada.

La Sociedad Española del Dolor (SED) y la Fundación Signo (FS) están promoviendo iniciativas conjuntas con la intención de desarrollar proyectos de investigación sobre mejoras organizativas y de gestión que aumenten la calidad, seguridad y eficiencia del tratamiento del dolor crónico. En este primer proyecto acometemos el análisis de la situación real y actualizada del tratamiento especializado del dolor crónico en España a través de un censo de los recursos, cartera de servicios e indicadores de calidad de las Unidades del Dolor, utilizando como guía el Documento de Estándares y Recomendaciones del Ministerio de Sanidad (MS) para las Unidades del Dolor $(12,13)$. Hemos recogido los datos mediante una encuesta nacional diseñada para que los facultativos puedan transmitir las características de las Unidades en base a su estructura, su dotación, su actividad, su cartera de servicios y la adaptación a los indicadores de calidad y buenas prácticas (12).

El análisis de los datos recogidos en la encuesta permitirá deducir la situación actual de las Unidades del Dolor, asumiendo como estándar los indicadores del documento publicado por el Ministerio de Sanidad (MS) y confrontando los datos recogidos con la tipología definida en el documento (tipo I: Unidad monográfica; tipo II: Unidad unidisciplinar para el tratamiento del dolor; y tipo III: Unidad multidisciplinar para el tratamiento del dolor).

De la lectura del documento editado por el MS; se deduce que el modelo ideal de Unidad de Dolor se aproxima al definido como Unidad Multidisciplinar para el Estudio y Tratamiento del Dolor de tipo III, que posee la siguiente dotación y características:

- Contar con un director, persona experta en el manejo de pacientes con dolor y con experiencia en investigación en dolor.

- Disponer de un espacio físico propio y adecuado para realizar sus actividades ambulatorias o en hospitalización.

- Estar en condiciones de tratar al paciente con dolor crónico de cualquier etiología, ofreciendo posibilidades diagnósticas y terapéuticas que abarquen tratamientos farmacológicos y no farmacológicos, incluyendo tratamientos invasivos de elevada complejidad y abordando aspectos médicos, psicológicos, sociales y laborales.

- Atender a un mínimo de 600 primeras visitas al año (para regiones sanitarias insulares o con población dispersa, basta considerar un mínimo de 400).

- Disponer, al menos, de dos especialistas médicos, uno de ellos de anestesiología o neurocirugía, de enfermeras y personal auxiliar y la colaboración de psicólogos, fisioterapeutas y trabajadores sociales.

- Realizar sesiones clínicas periódicas. 
- Elaborar una historia clínica electrónica del dolor que permita valorar la eficacia/eficiencia de los tratamientos.

- Disponer de protocolos propios y valorar su eficacia periódicamente.

\section{METODOLOGÍA}

Para elaborar un censo completo de las Unidades del Dolor en España que contenga la estructura, los recursos (humanos y materiales), la cartera de servicios, la actividad asistencial y poder analizar el cumplimiento de los estándares de calidad de cada una, se confeccionó una encuesta con los siguientes apartados:

- Datos de la unidad: denominación, centro y área donde está ubicada.

- Recursos humanos.

- Estructura y dotación.

- Datos de su actividad.

- Cartera de servicios.

- Utilización de indicadores de calidad y buenas prácticas.

Para que los resultados de la encuesta fueran fiables, la muestra analizada debería ser mayoritaria, o al menos con el número y la dispersión suficiente para garantizar que se trataba de una foto real de la situación de las Unidades de Dolor de España. Era necesario llegar al mayor número de facultativos responsables de estas Unidades, o en su caso a otros facultativos que trabajen en ellas, informarles de los objetivos, hacerles partícipes del proyecto y facilitarles el acceso a la encuesta. Para cumplir este objetivo hemos utilizado las fuentes de información y los recursos de comunicación accesibles desde la SED y la FS. Para los contactos, hemos trabajado con los datos existentes en los ficheros de la SED, la ayuda de los censos o ficheros de algunas sociedades autonómicas, capítulos de la SED, y otras fuentes aportadas desde las consejerías de salud, las gerencias de los hospitales públicos y algunos centros privados que fueron recabados por la FS. Todo ello se completó con datos existentes en el Ministerio de Sanidad, bien en el registro de las Unidades del Dolor, bien de forma indirecta mediante una deducción selectiva a partir del conjunto mínimo básico de datos (CMBD: registro que se recoge en España desde 1990, en cada hospital y por cada proceso y paciente, que refleja la actividad de cada centro hospitalario y su complejidad) de los Centros Sanitarios que nos brindó el propio MS.

La encuesta se hospedó en un sitio web específico, protegiendo su acceso mediante una palabra clave, y permaneció abierta para su cumplimentación desde el 6 de octubre de 2011 hasta el día 29 de febrero de 2012, fecha en que se cierra al contar con una muestra suficiente y que superaba las expectativas iniciales.

El sitio web que alojaba la encuesta disponía de un código de acceso que se proporcionó a todas las personas con- tactadas. Posteriormente, desde las secretarías de la SED o de la FS, se facilitó el acceso a los interesados acreditados que lo requirieron.

La encuesta se ofreció para su cumplimentación:

- A todos los socios de la SED, a los que se dirigió una carta personalizada solicitando su colaboración en el estudio. A todos ellos se les facilitó la clave de acceso a través de la hoja web de la sed: www.sedolor. es. En el momento de comenzar el proyecto, la SED tenía identificadas 147 unidades a las que también se solicitó por escrito su colaboración mediante carta individualizada.

- A todos los centros hospitalarios identificados en la base de datos de la FS y para los que se dejó abierta la encuesta en la hoja web de la Fundación Signo: www. fundacionsigno.com. Durante todo el periodo en que permaneció abierta la encuesta se registraron 23.831 visitas en esta web, pero no fue posible precisar los que correspondían a usuarios de la base de datos de hospitales. La base de datos de la Fundación recoge un total de 525 entidades, de las cuales 214 corresponden a hospitales, es decir, una amplia muestra del catálogo nacional de hospitales. La FS contactó con las gerencias y directores de estos centros para incentivar la cumplimentación de la encuesta.

- Se solicitó del Ministerio una relación de todos los Centros Sanitarios con su CMBD, para filtrar los que eran subsidiarios de disponer de una Unidad del Dolor y además una relación de los centros privados que tenían solicitada la licencia correspondiente para la apertura de una Unidad del Dolor.

Para conocer la tipificación de la Unidad se confeccionó una pregunta específica para identificar las Unidades con gestión independiente de otro servicio hospitalario.

Para identificar la dotación de recursos humanos se solicitó si la Unidad disponía de un coordinador o director y la descripción del personal que trabajaba a tiempo completo y con total dedicación, los que dedicaban sólo parte de su actividad a la Unidad, así como los que trabajan en ella sólo de forma esporádica.

Para identificar los procedimientos terapéuticos se utilizaron dos criterios:

- Cualitativo: para procedimientos realizados en hospital de día, quirúrgicos y psicoterapéuticos, considerando si están o no incluidos en la cartera de servicios de la Unidad.

- Cuantitativo: para el resto de los parámetros, referidos a la actividad realizada en 2010. Para los puntos característicos de distribución de cada una de las variables/ procedimientos, se utilizó el análisis de cuartiles, que corresponden al $25 \%, 50 \%$ y $75 \%$ de la muestra. Igualmente se ha considerado el porcentaje de exclusiones en cada uno de los procedimientos, es decir, Unidades que no lo realizan o no responden a la pregunta. 
Para validar la encuesta se realizaron ocho auditorías aleatorias de la muestra $(5,7 \%)$. Para la selección aleatoria se asignó un número secuencial a cada Unidad (según el orden de registro) y utilizando la función ALEATORIO()*141 de Excel 2007 se seleccionan ocho Unidades para realizar una auditoría de la encuesta cumplimentada por gestores de la FS.

Para comprobar los datos remitidos y verificarlos cuando existían incongruencias, de forma tangencial y paralela, se contactó con trabajadores de 74 de las Unidades censadas (el 52,48 \% de la muestra) para contrastar a distancia los datos remitidos (registrados o no registrados) mediante entrevista telefónica.

\section{RESULTADOS}

\section{Población e identificación de la muestra}

Se han analizado 141 encuestas una vez eliminados los duplicados. Las respuestas corresponden a 105 Unidades procedentes del registro de la SED (7 de ellas sin identificar, al reseñar de forma ambigua el centro donde se ubican: "Hospital" u "Hospital Universitario" sin que describan la población) y 36 Unidades que han accedido a la encuesta gracias a la información facilitada por la Fundación Signo y el Ministerio de Sanidad. El número de Unidades censadas aumentó desde las 147, conocidas previamente por la SED, a un total de 183, gracias a las identificadas por la aportación de la FS y el MS. Las 141 encuestas analizadas suponen un 80,3\% de la población total identificada, constituyendo una muestra suficiente y representativa.

TABLA I. DENOMINACIÓN FORMAL

\begin{tabular}{lcc}
\hline & Núm & $\%$ \\
\hline Unidad & 127 & 90,07 \\
Sin entidad organizativa propia & 14 & 9,93 \\
Total & 141 & 100,00 \\
\hline
\end{tabular}

TABLA II. ¿EN QUÉ SERVICIO O DEPARTAMENTO ESTÁ ENCUADRADA LA UNIDAD?

\begin{tabular}{lcc}
\hline & Núm & $\%$ \\
\hline Anestesiología & 117 & 82,98 \\
Gerencia o Dirección Médica & 22 & 15,60 \\
Reumatología & 1 & 0,71 \\
Autónoma & 1 & 0,71 \\
Total & 141 & 100,00 \\
\hline
\end{tabular}

El profesional que responde a la encuesta es mayoritariamente el director o coordinador de la Unidad $(64,96 \%)$. El resto, el 37,50 \%, ocupa una responsabilidad similar (médico adjunto, médico responsable, coordinador o jefe de servicio de anestesia, coordinador regional del dolor).

En cuanto a su denominación, el $90 \%$ de las encuestas aplican el nombre de Unidad del Dolor (Tabla I). El $83 \%$ estaban encuadradas en el Servicio de Anestesiología (Tabla II), el $83 \%$ tienen una antigüedad de más de cinco años (Tabla III). El $58 \%$ no asume la atención del dolor de otros hospitales y el $66 \%$ no atiende a población pediátrica.

\section{Recursos humanos}

En la mayoría de la Unidades existe un director o coordinador responsable del servicio (69\%). La mayoría a tiempo completo y sólo el $20 \%$ de ellas tienen un coordinador con dedicación exclusiva a la Unidad (Tabla IV). Cerca de un tercio de las Unidades del Dolor (31\%) no cuentan con responsable o director de la Unidad (Fig. 1).

La tabla $\mathrm{V}$ tipifica los profesionales que trabajan a tiempo completo en las Unidades (con una dedicación de 30 horas o más a la semana). El $55 \%$ dispone de uno o más anestesiólogos con esta dedicación. Otras especialidades con dedicación completa son: psicólogos (13,5\%), rehabilitadores (9\%), médicos de familia (7\%), internistas (6\%), neurólogos $(3,5 \%)$, psiquiatras $(3 \%)$ y neurocirujanos $(2 \%)$. El $4 \%$ de las Unidades declaran otras especialidades (geriatría y otras). El 57\% de las Unidades dispone de una o más enfermeras, el $30 \%$ cuenta con auxiliares de

TABLA III. ANTIGÜEDAD DE LA UNIDAD

\begin{tabular}{lcc}
\hline & Núm & $\%$ \\
\hline Menos de 1 año & 4 & 2,84 \\
De 1 a 5 años & 20 & 14,18 \\
Más de 5 años & 117 & 82,98 \\
Total & 141 & 100,00 \\
\hline
\end{tabular}

TABLA IV. ¿EXISTE UN DIRECTOR O COORDINADOR DE LA UNIDAD?

\begin{tabular}{lcc}
\hline & Núm & $\%$ \\
\hline Sí, con dedicación exclusiva & 28 & 19,86 \\
Sí, a tiempo completo & 69 & 48,94 \\
No & 44 & 31,21 \\
Total & 141 & 100,00 \\
\hline
\end{tabular}


enfermería, el $6 \%$ con fisioterapeutas y el $21 \%$ tiene auxiliares administrativos. Sólo una Unidad declara disponer de un trabajador social a tiempo completo.

Sobre el personal que participa a tiempo parcial en la Unidad (profesionales que trabajan en la Unidad de forma habitual, más de 10 horas a la semana y menos de 30), el $67 \%$ son anestesiólogos, el $12 \%$ psicólogos, un $10 \%$ neurocirujanos y otro $10 \%$ rehabilitadores. Además, el $26 \%$ de las Unidades dispone de una o más enfermeras a que trabajan a tiempo parcial, el $16 \%$ auxiliares administrativos, el $12 \%$ celadores y el $10 \%$ auxiliares de enfermería.

De las Unidades que registraron no tener ninguna persona a tiempo parcial, 22 declaran tener personal con menos de 10 horas semanales de dedicación. Hay 10 Unidades que refieren otro tipo de contratación más esporádica al registrado en esta pregunta. En ambos casos los trabajadores esporádicos son mayoritariamente anestesiólogos.

\section{Dotación de la Unidad}

La mayoría de las Unidades (70 \%) declaran no disponer de camas propias, bien porque responden negativamente o bien porque no responden a la pregunta. El $18 \%$ de las Unidades declaran 1 o 2 camas propias y el $12 \%$ más de dos camas.

El $63 \%$ no refiere actividad quirúrgica. El $23 \%$ de las Unidades refieren una sesión quirúrgica semanal de 7 horas, el $9 \%$ dos sesiones semanales y el $6 \%$ más de tres.

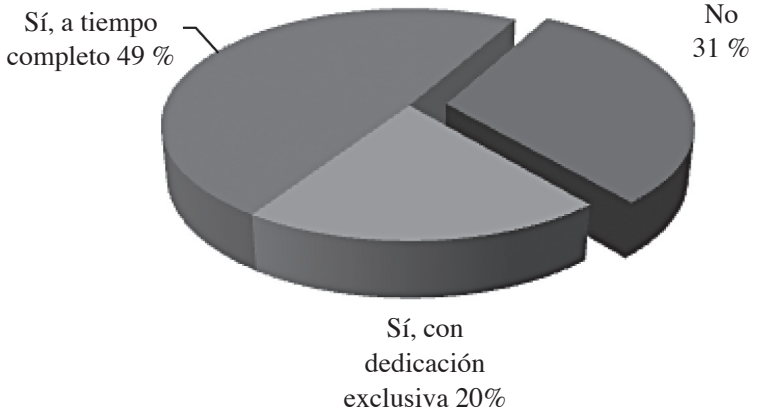

Fig. 1. ¿Existe un Director o Coordinador de la Unidad?

El $52 \%$ de las Unidades expresan que no disponen de salas de técnicas de bloqueo de uso propio, bien porque realmente no cuentan con ellas o bien porque no responden. El $20 \%$ dispone de una, el $16 \%$ entre dos y cuatro y el $12 \%$ cinco o más.

El $69 \%$ de las Unidades indican tener entre uno y tres despachos de consulta propios, el $12 \%$ dispone de cuatro a seis despachos y el $19 \%$ no tienen local propio, bien porque realmente no cuentan con él o bien porque no responden. En otra pregunta, el $34 \%$ de las Unidades refieren que utilizan para la consulta despachos compartidos con otro servicio del hospital.

El $42 \%$ de las Unidades no disponen de camas de hospital de día propias. El $43 \%$ dispone entre una y cuatro camas/puestos de hospital de día, un $9 \%$ tiene cinco o seis y el $6 \%$ restante refiere siete o más.

TABLA V. PERSONAS A TIEMPO COMPLETO. \% DE UNIDADES CON...

\begin{tabular}{lcccccc}
\hline & 1 & 2 & 3 & 4 & 5 o más & Total \\
\hline Anestesiólogos & 30,5 & 16,3 & 2,8 & 2,8 & 2,1 & 54,6 \\
Neurocirujanos & 1,4 & 0,7 & & & & 2,1 \\
Rehabilitadores & 9,2 & & & & & 9,2 \\
Medicina Interna & 5,0 & 0,7 & & & 5,7 \\
Neurólogos & 3,5 & & & & & 3,5 \\
Psiquiatras & 2,8 & & & & & 2,8 \\
Psicólogos & 12,1 & 1,4 & & & 13,5 \\
Otra especialidad & 2,8 & 1,4 & & & 4,3 \\
Médicos de familia & 2,8 & 2,1 & 1,4 & & 0,7 & 7,1 \\
Enfermeras & 39,0 & 15,6 & & 1,4 & 1,4 & 57,4 \\
Auxiliares de enfermería & 26,2 & 3,5 & & & 29,8 \\
Fisioterapeutas/terapeutas ocupacionales & 2,8 & 1,4 & 1,4 & & & 5,7 \\
Trabajadores sociales & 0,7 & & & & & 0,7 \\
Auxiliares administrativos & 19,9 & 1,4 & & & & 21,3 \\
Celadores & 8,5 & & & & & 8,5 \\
\hline
\end{tabular}


Rev. Soc. Esp. del Dolor, Vol. 21, N. ${ }^{\circ}$ 3, Mayo-Junio 2014

El $75 \%$ de las Unidades no dispone de salas de terapia de grupo o no responde a la pregunta. El $24 \%$ dispone de una y una Unidad refiere tener cuatro.

\section{Actividad}

El $57 \%$ de las Unidades no registran altas de hospitalización o no responden a esta pregunta. Del $43 \%$ restante, un tercio (el $39 \%$ ) había declarado no disponer de camas propias de hospitalización en la cuestión correspondiente. Los cuartiles de la muestra se sitúan en 10, 29 y 60 (Tabla VI).

Sobre el índice de ocupación, el $69 \%$ de las Unidades, o no responde a esta pregunta o no conoce el índice de ocupación de sus camas durante el año 2010. De los que responden (31\% de la muestra), en la mitad de ellos (48\%) su respuesta no se corresponde con el número de camas (o bien no han declarado camas propias o su número no coincide con el calculo del índice de ocupación), por lo que sólo fue valorable el $18 \%$ de la muestra. Aún así, hemos evaluado los datos aportados y los cuartiles de la muestra se sitúan en 25,50 y $86 \%$. Sucede lo mismo con la estancia media: el $62 \%$ no responde, no conoce el dato o no lo registra; del $38 \%$ restante, en casi la mitad de ellos (44\%) el dato aportado no corresponde con el número de camas y en el resto (20\% de la muestra total) los cuartiles se sitúan en 1,$51 ; 2,32$ y 4,99. En cuanto a la pregunta sobre el peso medio del grupo relacionado con el diagnóstico (GRD: clasificación y agrupación de los pacientes atendidos en los centros sanitarios según su diagnóstico) al alta, se dejó como respuesta voluntaria ya que se desconocía por la generalidad de los encuestados y podía desanimar a cumplimentar la encuesta. Sólo respondieron el $11 \%$ de las Unidades; la mitad declararon un peso medio entre 1,64 y 4,25 (e incluso más de 4,25), que parece indicar que el peso medio se ha recogido del alta de procesos globales en los que la Unidad había participado.

El número de primeras visitas a las Unidades se sitúan en los cuartiles 300, 583, 806. El $7 \%$ no respondieron o no registran esta actividad (Tabla VII).

TABLA VI. NÚMERO DE ALTAS DE HOSPITALIZACIÓN (ACTIVIDAD 2010)

\begin{tabular}{lcc}
\hline & Núm. & $\%$ \\
\hline Menos de 10 & 11 & 7,80 \\
Entre 10 y 29 & 19 & 13,48 \\
Entre 30 y 60 & 15 & 10,64 \\
Más de 60 & 16 & 11,35 \\
NS/NC & 80 & 56,74 \\
Total & 141 & 100,00 \\
\hline
\end{tabular}

TABLA VII. NÚMERO DE PRIMERAS VISITAS (ACTIVIDAD 2010)

\begin{tabular}{lcc}
\hline & Núm. & $\%$ \\
\hline Menos de 300 & 31 & 21,99 \\
Entre 300 y 583 & 34 & 24,11 \\
Entre 584 y 806 & 33 & 23,40 \\
Más de 806,5 & 33 & 23,40 \\
NS/NC & 10 & 7,09 \\
Total & 141 & 100,00 \\
\hline
\end{tabular}

Los cuartiles para el número de visitas sucesivas se sitúan en $905,1.649$ y 3058 . El $7 \%$ de la muestra no responden o no identifican esta actividad (Tabla VIII). Las ratio sucesivas/primeras para esos mismos cuartiles se cifra en 3,02; 2,83 y 3,79.

\section{Procedimientos}

Los procedimientos no quirúrgicos, realizados en las salas de técnicas o despachos de las Unidades, están incluidos en la mayoría ( $80 \%$ ) de las carteras de servicios de las Unidades y quedan reflejadas en la figura 2.

Ninguno de los procedimientos está por debajo del $20 \%$ de utilización, aunque se citan con menos frecuencia la infusión intravenosa con bifosfonatos y el test intravenoso con fentolamina.

Otros procedimientos que se realizan en quirófano se enumeran en la tabla IX y forman parte de la mayoría (78\%) de la cartera de servicios de las Unidades.

Por debajo del $20 \%$ se encuentran: el tratamiento intradiscal electrotérmico (IDET), vertebroplastia/cifoplastia, epiduroscopia, ozonoterapia y discografía.

Algunos procedimientos quirúrgicos de frecuencia media que están incluidos en la cartera de servicios de la mitad de las Unidades (cerca del 50 \%) como la estimulación medular y el implante de bombas subcutáneas, coinciden

TABLA VIII. NÚMERO DE VISITAS SUCESIVAS (ACTIVIDAD 2010)

\begin{tabular}{lcc}
\hline & Núm. & $\%$ \\
\hline Menos de 905 & 33 & 23,40 \\
Entre 905 y 1.649 & 32 & 22,70 \\
Entre 1.650 y 3.057 & 33 & 23,40 \\
Más de 3.058 & 33 & 23,40 \\
NS/NC & 10 & 7,09 \\
Total & 141 & 100,00 \\
\hline
\end{tabular}


con las Unidades mejor dotadas, constituyendo un carácter diferencial positivo (Tablas X y XI).

Un procedimiento muy generalizado en las Unidades (66\%) es la neurólisis mediante radiofrecuencia. La encuesta refleja que la mayoría de las sesiones quirúrgicas de las Unidades se utilizan para aplicar este tratamiento (Tabla XII).

El $72 \%$ de las Unidades no realizó ningún procedimiento psicoterapéutico durante 2010 o bien no respondieron a esta cuestión. Para el $28 \%$ de las que lo utilizan, los cuartiles se sitúan en 15, 60 y 117. El procedimiento psicoterapéutico que más se realiza es el tratamiento cognitivo-conductual (37\%) y el que menos $(8 \%)$ las sesiones de biofeedback y la hipnosis.

\section{Buenas prácticas}

En cuanto al grado de implantación de buenas prácticas, más del $85 \%$ de las Unidades refieren tener implantado "un modelo de consentimiento del enfermo para procedimientos que impliquen riesgo, con información particularizada para cada uno", el $63 \%$ declaran que "se realizan reuniones periódicas del equipo de la Unidad para analizar los incidentes de seguridad", pero el resto de los indicadores de calidad tienen una muy escasa, casi nula, implantación.

\section{AUDITORÍA Y VALIDACIÓN DEL CENSO}

El objetivo de las auditorías ha sido estimar la veracidad de la encuesta: "Censo de los recursos, cartera de servicios y análisis sistemático de los indicadores de las Unidades del Dolor", a través de la verificación del grado de coincidencia

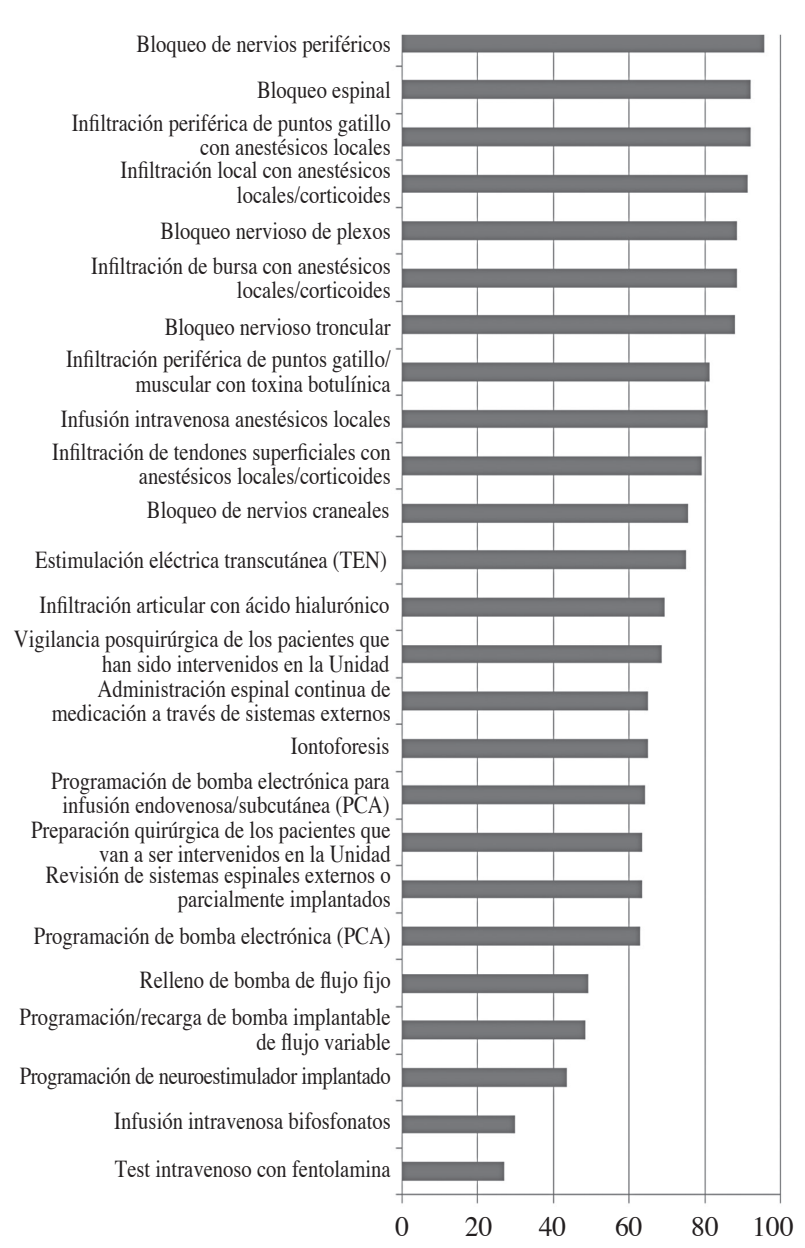

Fig. 2. Procedimientos en Sala de Bloqueos/Hospital de Día, ordenados de mayor a menor utilización.

TABLA IX. PROCEDIMIENTOS QUIRÚRGICOS

\begin{tabular}{|c|c|c|c|}
\hline Procedimiento & $\%$ Sí & $\%$ No & $\% N S / N C$ \\
\hline $\begin{array}{l}\text { Técnicas de punción muscular profunda con control radiológico: toxina botulínica de } \\
\text { psoas/cuadrado lumbar/piriforme }\end{array}$ & 79,4 & 20,6 & \\
\hline Bloqueo de ganglio esfenopalatino & 44,7 & 55,3 & \\
\hline Bloqueo simpático cérvico-torácico diagnóstico o terapéutico (ganglio estrellado) & 78,0 & 22,0 & \\
\hline Bloqueo neurólisis paravertebral simpático & 53,9 & 46,1 & \\
\hline Bloqueo neurolítico, epidural o subaracnoideo & 56,0 & 44,0 & \\
\hline Bloqueo sacroiliaco & 83,7 & 16,3 & \\
\hline Bloqueo coxofemoral & 78,7 & 21,3 & \\
\hline Bloqueo/neurólisis del plexo celiaco & 66,0 & 34,0 & \\
\hline Bloqueo/ neurólisis del plexo hipogástrico superior & 51,1 & 48,9 & \\
\hline Bloqueo facetario/ramo medial posterior vertebral: cervical/dorsal/lumbar & 87,9 & 12,1 & \\
\hline $\begin{array}{l}\text { Bloqueo radicular selectivo de raíces espinales (inyección transforaminal dorsal, lumbar } \\
\text { y sacra) }\end{array}$ & 75,2 & 24,8 & \\
\hline
\end{tabular}


TABLA IX. (Cont.) PROCEDIMIENTOS QUIRÚRGICOS

\begin{tabular}{|c|c|c|c|}
\hline Procedimiento & $\%$ Si & $\%$ No & $\% N S / N C$ \\
\hline Discografía & 19,1 & 80,9 & \\
\hline Tratamiento intradiscal electrotérmico (IDET) & 5,0 & 95,0 & \\
\hline Catéter espinal tunelizado con/sin bomba de infusión externa & 78,7 & 21,3 & \\
\hline Cordotomía cervical percutánea & 5,0 & 95,0 & \\
\hline Epidurolisis/adhesiolisis por adherencias & 41,1 & 58,9 & \\
\hline Epiduroscopia & 14,9 & 85,1 & \\
\hline Radiofrecuencia de articulaciones vertebrales posteriores (ramo medial) & 66,7 & 33,3 & \\
\hline Radiofrecuencia de nervios periféricos & 66,0 & 34,0 & \\
\hline Radiofrecuencia de cadena simpática cervical, torácica, lumbar & 51,8 & 48,2 & \\
\hline Radiofrecuencia de ganglio dorsal: cervical, dorsal o lumbar & 58,9 & 41,1 & \\
\hline Radiofrecuencia de raíces posteriores vía epidural & 48,9 & 51,1 & \\
\hline Radiofrecuencia del disco intervertebral & 22,0 & 78,0 & \\
\hline Radiofrecuencia y otras intervenciones terapéuticas sobre el ganglio de Gasser & 39,7 & 60,3 & \\
\hline Radiofrecuencia de nervios esplácnicos & 34,0 & 66,0 & \\
\hline Radiofrecuencia de articulación sacroiliaca & 54,6 & 45,4 & \\
\hline Radiofrecuencia de ganglio impar de Walter & 55,3 & 44,7 & \\
\hline Radiofrecuencia intraarticular coxofemoral y de rodilla & 46,8 & 53,2 & \\
\hline Implantes de electrodos subcutáneos, periféricos y radiculares & 34,8 & 65,2 & \\
\hline Implante de electrodos de estimulación medular & 49,6 & 50,4 & \\
\hline Implante de electrodo quirúrgico epidural & 27,0 & 73,0 & \\
\hline Recolocación/sustitución o retirada de electrodos de estimulación medular (Q) & 47,9 & 51,4 & 0,7 \\
\hline Implante de generador interno y extensión a electrodos ( $2 .^{\circ}$ tiempo) & 48,6 & 50,7 & 0,7 \\
\hline Sustitución de generador interno & 47,9 & 51,4 & 0,7 \\
\hline Implante revisión y/o recambio de bomba de infusión interna & 47,1 & 52,2 & 0,7 \\
\hline $\begin{array}{l}\text { Implante, revisión y/o recambio de reservorio para infusión intratecal lumbar, dorsal o } \\
\text { cervical }\end{array}$ & 50,7 & 48,6 & 0,7 \\
\hline Vertebroplastia/cifoplastia & 12,1 & 87,2 & 0,7 \\
\hline Bloqueo ganglio Walter & 64,3 & 35,0 & 0,7 \\
\hline Ozonoterapia: infiltración y discólisis & 16,5 & 82,1 & 1,4 \\
\hline
\end{tabular}

entre las respuestas a la encuesta recogidas en la web, y las obtenidas "in situ" realizadas a ocho Unidades seleccionadas aleatoriamente $(5,7 \%)$ de la muestra. Los datos se verifican por dos vías; por un lado con el responsable de la Unidad y por otro, con la Dirección/Gerencia del Hospital. Adicionalmente se ha realizado una "comprobación a distancia" (mediante entrevista telefónica) de la información recogida desde 74 Unidades $(52,48 \%$ de la muestra).
El "grado de coincidencia" se ha evaluado para los siguientes apartados:

1. Datos de la Unidad.

2. Recursos humanos de la Unidad.

3. Dotación de la Unidad.

4. Actividad de la Unidad.

5. Procedimientos de la Unidad.

6. Buenas prácticas. 
TABLA X. ESTIMULACIÓN MEDULAR (NÚMERO EN 2010)

\begin{tabular}{lcc}
\hline & Núm. & $\%$ \\
\hline Menos de 4 & 13 & 9,22 \\
Entre 4 y 9 & 18 & 12,77 \\
Entre 10 y 14 & 13 & 9,22 \\
Más de 14 & 19 & 13,48 \\
NS/NC & 78 & 55,32 \\
Total & 141 & 100,00 \\
\hline
\end{tabular}

TABLA XI. INFUSIÓN INTRATECAL (NÚMERO EN 2010)

\begin{tabular}{lcc}
\hline & Núm. & $\%$ \\
\hline Menos de 2 & 18 & 12,77 \\
Entre 2 y 3 & 17 & 12,06 \\
Entre 4 y 9 & 21 & 14,89 \\
Más de 9 & 24 & 17,02 \\
NS/NC & 61 & 43,26 \\
Total & 141 & 100,00 \\
\hline
\end{tabular}

El mayor grado de coincidencia se obtiene en el registro de actividad (100\%), la definición de la cartera de servicios/procedimientos (95\%), los datos de identificación de la Unidad (94\%) y la dotación (94\%).

Los peores se muestran en el registro de los recursos humanos de la Unidad (72\%) y en la identificación de las buenas prácticas $(75 \%)$. En el conjunto el grado de coincidencia supera el $81,4 \%$.

El 18,6\% global de discrepancia entre la auditoría y el censo no sigue una regla común, por ejemplo, en el sentido de haber declarado cifras "superiores" a las reales. Se ha podido verificar que en muchos casos se trata de errores de interpretación y/o de introducción, excepto en el caso de la Unidad número 2, donde circunstancias excepcionales y por tanto difícilmente reproducibles en otras Unidades, la sitúan en un grado de coincidencia inferior al $50 \%$.

Eliminando la Unidad 2 como extremo no representativo, el grado de coincidencia supera el $86 \%$, que es el valor medio de veracidad que consideramos para este primer Censo de la Unidades del Dolor en España, con un intervalo de confianza que se sitúa entre el 81 y el $90 \%$.

\section{DISCUSIÓN}

El tamaño de la muestra y el grado de coincidencia entre los datos obtenidos por los auditores y los aportados por
TABLA XII. RADIOFRECUENCIA (NÚMERO EN 2010)

\begin{tabular}{lcc}
\hline & Núm. & $\%$ \\
\hline Menos de 46 & 22 & 15,60 \\
Entre 46 y 99 & 21 & 14,89 \\
Entre 100 y 224 & 23 & 16,31 \\
Más de 224 & 23 & 16,31 \\
NS/NC & 52 & 36,88 \\
Total & 141 & 100,00 \\
\hline
\end{tabular}

TABLA XIII. CLASIFICACIÓN DE LAS UNIDADES DEL CENSO POR NIVELES DEL DOCUMENTO DE ESTÁNDARES Y RECOMENDACIONES

\begin{tabular}{lcc}
\hline \multicolumn{1}{c}{ Nivel } & Totales & $\%$ \\
\hline I. Unidad monográfica & 0 & 0,0 \\
II. Unidad unidisciplinaria & 119 & 84,4 \\
III. Unidad multidisciplinaria & 6 & 4,3 \\
No clasificada & 16 & 11,3 \\
Totales & 141 & 100,0 \\
\hline
\end{tabular}

las Unidades del Dolor, demuestran que las características recogidas reflejan fielmente la realidad. No deja de extrañar, una vez más, que el grado de coincidencia sea menor en el parámetro de recursos humanos de las Unidades, con una clara tendencia a la inflación del dato real, lo cual no favorece el rendimiento resultante de las Unidades.

La mayoría de los encuestados (90 \%) utilizan la denominación de "Unidad del Dolor", sea cual sea su estructura, y no es infrecuente que se aplique este nombre a simples consultas del dolor compartidas con otros servicios (34\%), que no cumplen los mínimos necesarios para tal denominación y otras $(63 \%)$ que, al no realizar técnicas quirúrgicas (radiofrecuencia...), son claramente insuficientes para dar respuesta a la problemática de los pacientes complejos, aunque debemos reconocer que unas y otras cumplen un soporte básico y esencial en nuestra especialidad. Por tanto, no podemos ni debemos ignorar su realidad, pero para ponderar el colectivo es necesario tipificarlas y poder diferenciarlas de Unidades mejor dotadas. Si aplicamos los datos recogidos en la encuesta a la tipología según las define el Documento de Estándares y Recomendaciones del Ministerio de Sanidad, el $0 \%$ de las Unidades se encuadran en el tipo I, mientras que el $85 \%$, es decir, la gran mayoría de las Unidades censadas, se encuadran en el tipo II que corresponde a Unidades unidisciplinarias (Tabla XIII).

Esta adaptación a la clasificación editada por el Ministerio (que se basa en la editada por la IASP, adaptada a la 
Rev. Soc. Esp. del Dolor, Vol. 21, N. ${ }^{\circ}$ 3, Mayo-Junio 2014

situación en EE. UU. y no a España), agrupa en el nivel II casi la totalidad de las Unidades españolas (Tabla XIII), desde las consultas compartidas hasta Unidades perfectamente dotadas con varios facultativos con dedicación total, por lo que la clasificación pierde validez y sentido.

Con intención de estratificar las denominadas Unidades unidisciplinarias (que definen el $85 \%$ de todas las existentes) y sólo para poder sacar conclusiones de los resultados de este trabajo, diferenciando unas de otras según sus recursos y su actividad, las hemos agrupado de la siguiente manera:

- Grupo A: sólo 1 médico (especialista o no) a tiempo parcial, que no pasa consulta diaria.

- Grupo B: Unidades que disponen de 1 especialista a tiempo parcial y consulta diaria.

- Grupo C: Unidades que disponen de 1 especialista a tiempo completo y consulta diaria.

- Grupo D: Unidades con más de 1 médico a tiempo completo y consulta diaria.

Los resultados obtenidos se muestran en la tabla XIV. Eliminando las Unidades unidisciplinarias que no se pueden asignar a ningún grupo por falta de datos (11\%), el resto (89\%) lo hemos agrupado de la siguiente manera: el $25 \%$ corresponderían a Unidades unidisciplinarias con un especialista a tiempo parcial y que no pasan consulta diariamente (grupo A). El $19 \%$ corresponde a Unidades unidisciplinarias con un especialista a tiempo parcial y consulta diaria (grupo B) y el $33 \%$ son Unidades unidisciplinarias con un especialista a tiempo completo y consulta diaria (grupo C). El resto, (8\%) son Unidades unidisciplinarias que cuentan con más de un médico (de la misma especialidad) a tiempo completo (grupo D).

Es de reseñar que un tercio de las Unidades carezcan de un director o responsable y sin estructura organizativa. Esto puede ser el origen de un grave problema tanto para la dotación de recursos, la dedicación e incentivación de los profesionales y la continuidad de la Unidad como para la calidad de la asistencia.

La gran mayoría de las Unidades censadas tienen una antigüedad superior a 5 años, lo que constituye un dato po- sitivo en cuanto a la fiabilidad y seguridad del tratamiento (14). También conviene destacar que el $83 \%$ de las Unidades dependen de los Servicios de Anestesiología, lo cual entraña una dificultad importante para su evolución hacia Unidades de gestión independiente, como aconseja el Documento del Ministerio (12). Es de reseñar que las Unidades independientes tienen mayores índices de complejidad (100\% son de nivel III) y están entre las mejor dotadas, constituyendo un carácter diferencial positivo (sólo un $2 \%$ de las Unidades dependientes de otro servicio, generalmente Anestesia, son de nivel III).

En cuanto a las especialidades, como era de esperar, la anestesiología sigue constituyendo la base en la que se sustenta el tratamiento del dolor. Irrumpen otros especialistas que tienen una presencia notable, como la rehabilitación que ocupa el $10 \%$ de la plantilla de los facultativos de las Unidades. También llama la atención de forma positiva la presencia de un número creciente de psicólogos, aunque el 13,5\% sigue siendo muy inferior a la mínima deseable (15).

Uno de los datos que no fueron bien registrados por los encuestados es el número de camas instaladas (asignadas) a la Unidad del Dolor. Este dato sirve para obtener los índices de ocupación y estancia media. Si no existen camas asignadas a la Unidad no es posible calcular estos índices. Sólo el $31 \%$ aporta estas cifras y en la mitad de ellos los datos aportados no se corresponden con las camas declaradas en la encuesta. Creemos que no se entendió la pregunta, por lo que consideramos que estos resultados (válidos sólo en el $18 \%$ de los encuestados) no aportan valor a la encuesta, por lo que los hemos ignorado en nuestras conclusiones.

Sin embargo, es muy significativo que la cartera de procedimientos, sobre todo la actividad quirúrgica, sea claramente descriptiva de la complejidad de la Unidad, siendo las Unidades que refieren mayor dotación en recursos humanos y estructura, las que contienen carteras con mayor número y variedad de procedimientos (Tabla XV).

La radiofrecuencia es el procedimiento más extendido (la mayoría de las técnicas quirúrgicas realizadas, excluyendo implantes) y no aporta carácter discriminativo entre

TABLA XIV. CLASIFICACIÓN DE LAS UNIDADES DEL CENSO POR NIVELES DE ACTIVIDAD Y CATEGORÍAS DE DISPONIBILIDAD (EN \%)

\begin{tabular}{lccccc}
\hline \multicolumn{7}{c}{ Nivel según Ministerio de Sanidad } & $A$ & $B$ & $C$ & $D$ & \% Muestra \\
\hline I. Unidad monográfica & $\ldots$ & $\ldots$ & $\ldots$ & $\ldots$ & 0,0 \\
II. Unidad unidisciplinaria & 24,8 & 19,1 & 32,6 & 7,8 & 84,4 \\
III. Unidad multidisciplinaria & --- & --- & --- & --- & 4,3 \\
No clasificada & --- & --- & --- & -- & 11,3 \\
\% sólo Unidades unidisciplinares & 29,4 & 22,7 & 38,6 & 9,2 & 100 \\
\hline
\end{tabular}


TABLA XV. PORCENTAJE DE UNIDADES UNIDISCIPLINARES (NIVEL II) QUE REALIZAN UN TIPO DE TRATAMIENTO (EN \%)

\begin{tabular}{lccccc}
\hline & \multicolumn{5}{c}{ Unidades unidisciplinares (nivel II) } \\
\hline \multicolumn{1}{c}{ Tratamientos empleados } & $A$ & $B$ & $C$ & $D$ & Global \\
\hline Farmacológico & $100 \%$ & $100 \%$ & $100 \%$ & $100 \%$ & $100 \%$ \\
Bloqueos periféricos/infiltraciones & --- & $37 \%$ & $100 \%$ & $100 \%$ & $56 \%$ \\
Bloqueos centr./tec. no quirúrg. & --- & $14,8 \%$ & $67,4 \%$ & $100 \%$ & $39 \%$ \\
Técnicas quirúrgicas (no implante) & --- & --- & $67,4 \%$ & $100 \%$ & $35 \%$ \\
Técnicas de implante & --- & --- & $8,7 \%$ & $100 \%$ & $13 \%$ \\
Psicoterapia & --- & $7,4 \%$ & $8,7 \%$ & $90,2 \%$ & $13 \%$ \\
\hline
\end{tabular}

las Unidades C y D. Los procedimientos de estimulación medular y la infusión espinal (técnicas de implante) contienen carácter discriminatorio positivo al estar incluidos esencialmente en las carteras de procedimientos de las Unidades mejor dotadas.

En cuanto al registro de calidad y buenas prácticas, es reseñable que la gran mayoría de las Unidades no hacen seguimiento de estos indicadores y con la excepción del consentimiento informado para procedimientos de riesgo, utilizado casi universalmente por sus connotaciones legales, no se sigue ningún otro protocolo salvo excepciones.

El resultado de la auditoría aleatoria, que se realizó en un $5,7 \%$ de los centros que respondieron, es representativo de la muestra con altos índices de coincidencia entre las respuestas de la encuesta y las recogidas por los auditores. El mayor grado de coincidencia se muestra en la actividad de la Unidad y la cartera de servicios y el mayor grado de discrepancia en los recursos humanos, siempre con tendencia a la inflación. La discrepancia en los otros apartados no sigue una regla común, en el sentido de haber declarado cifras "superiores" a las reales, sino que los auditores han detectado muchos errores de interpretación. Solamente una Unidad auditada mostró grado de coincidencia muy inferior al $50 \%$ por circunstancias excepcionales y no reproducibles por lo que se eliminó como extremo no representativo. Con ello el grado de coincidencia supera el $86 \%$, que es el valor medio de veracidad de la encuesta con intervalo de confianza del $81-90 \%$ y que valida suficientemente los resultados.

\section{CONCLUSIONES}

Si se tipifican las Unidades según la dotación declarada en las encuestas, y se compara con la cartera de servicios y su actividad, se pone de manifiesto que las peor dotadas y que disponen de menos recursos muestran menores alternativas terapéuticas. Las Unidades que cuentan con especialistas que dedican sólo parte de su actividad al tra- tamiento del dolor, reflejan en sus datos una actividad asistencial poco resolutiva frente al paciente con dolor crónico complejo. Si además la Unidad no dispone de una atención diaria, las carencias empeoran de forma grave. Esta situación es radicalmente diferente a la que ocurre en otras Unidades mejor dotadas, con consulta diaria, que cuentan con, al menos, un especialista con dedicación completa al tratamiento del dolor. En el vértice de la pirámide estarían las Unidades que cuentan con dos o más especialistas con dedicación exclusiva a la Unidad y que ofrecen atención continua, cubriendo el cuidado de los enfermos los días vacacionales o de ausencia forzada y además asumiendo procedimientos (quirúrgicos o bloqueos) sin abandonar la actividad de consulta. Por tanto, no es banal la estratificación de las Unidades, ya que refleja una capacidad terapéutica distinta, desde las menos dotadas hasta las más cualificadas, seguras y eficaces. El cajón de sastre actual que agrupa el $83 \%$ de las Unidades en Unidades de tipo II, todas iguales, agrupando en el mismo tipo a Unidades que atienden a los pacientes de forma parcial, sin estructura propia ni recursos materiales, con otras Unidades que tienen varios facultativos de la misma disciplina, con dedicación total y que realizan todo tipo de técnicas, incluidas las más sofisticadas para el tratamiento del dolor, debería revisarse. Entendemos que distinguir entre las distintas Unidades según sus capacidades es útil para objetivar la situación, denunciar deficiencias y reclamar planes de mejora.

Además, consideramos que se debe modificar la definición actual de Unidades de tipo I, ya que no existen en España. En cuanto a las Unidades multidisciplinares de tipo III, aunque por su pequeño número parece menos necesario estratificarlas, considerando su crecimiento futuro y deseable, hemos abordado también una propuesta de su subclasificación.

En síntesis, al percibir con los datos de la encuesta que las definiciones y clasificación de las Unidades del Dolor establecidas en el Documento de Estándares de Calidad del Ministerio de Sanidad no se adaptan a la realidad de 
Rev. Soc. Esp. del Dolor, Vol. 21, N. ${ }^{\circ}$ 3, Mayo-Junio 2014

nuestro país, consideramos que sería necesario abordar la confección de una nueva clasificación de las Unidades. Tras un concienzudo análisis de la situación actual, proponemos para su consideración al comité de acreditación de la SED las siguientes definiciones y clasificación:

- Unidades tipo I: Unidades que disponen de uno o varios especialistas con dedicación a tiempo parcial a la Unidad y que no dispensan atención continua diaria. Con esta nueva definición, el tipo I albergaría al $25 \%$ de las Unidades españolas. Según la encuesta (grupo A), en la mayoría de estas Unidades sólo se administra tratamiento farmacológico; en alguna de ellas se realiza esporádicamente alguna técnica simple (bloqueo o infiltración).

- Unidades tipo II:

- Subtipo (a): Unidades que disponen de uno o varios especialistas, con dedicación a tiempo parcial a la Unidad y que dispensan atención continua y diaria.

Albergaría el $20 \%$ de las Unidades españolas actuales. En la mayoría de estas Unidades se administra fundamentalmente tratamiento farmacológico y con cierta frecuencia técnicas simples de bloqueo o infiltración.

- Subtipo (b): Unidades con un solo especialista, con dedicación a tiempo completo y que dispensa atención continua y diaria.

Cumplen estos criterios el $32 \%$ de las Unidades españolas, siendo sin duda el tipo más frecuente. En la mayoría de estas Unidades además del tratamiento farmacológico se realizan técnicas de bloqueos/ infiltración, bloqueos con radiofrecuencia y algunas técnicas quirúrgicas de implante.

- Unidades tipo III: Unidades que disponen de más de un médico especialista, de la misma disciplina, todos con dedicación a tiempo completo y que dispensan atención continua y diaria.

Actualmente cumplen estos criterios el $8 \%$ de las Unidades españolas. En estas Unidades, además del tratamiento farmacológico se realizan todo tipo de técnicas de bloqueos/infiltración, bloqueos con radiofrecuencia y técnicas quirúrgicas de implante.

- Unidades multidisciplinares tipo IV: Unidades que disponen de más de un médico especialista, de al menos dos disciplinas distintas, todos con dedicación a tiempo completo y que dispensan atención continua $y$ diaria.

Actualmente cumplen estos criterios el $4 \%$ de las Unidades. En la mayoría de estas Unidades además del tratamiento farmacológico se realiza gran número de técnicas de bloqueos/infiltración, bloqueos con radiofrecuencia y técnicas quirúrgicas de implante.

- Subtipo (a): no cuentan con psicólogo como plantilla de la Unidad y a tiempo completo.
- Subtipo (b): cuentan con psicólogo a tiempo total, con consulta diaria, integrado en la plantilla de la Unidad.

Finalmente cabría plantearse cuál es la distribución de los recursos existentes en la actualidad y cuáles serían los mínimos exigibles para cubrir mínimamente las necesidades sanitarias de la población española en cuanto a la atención y el tratamiento del dolor crónico. Con estos datos, podríamos sentar las bases de una reflexión seria sobre los mejora de nuestros recursos, cubrir las deficiencias que han quedado manifiestas en este trabajo y determinar hacia dónde se deben dirigir los esfuerzos para cumplir con los estándares de calidad que establece el documento del Ministerio de Sanidad.

Es reseñable el pobre registro y el nulo seguimiento de los ítems de calidad y buenas prácticas, que mejorarían con el esfuerzo de todos los directores y responsables de las Unidades del Dolor, y que aumentarían la fiabilidad y la categoría científica, sanitaria y administrativa de nuestras Unidades.

\section{AGRADECIMIENTOS}

Este trabajo ha sido tutorizado por la Sociedad Española del Dolor y para su realización se ha contado con la colaboración de la Fundación Signo y el apoyo económico y desinteresado de la Fundación Grünenthal.

\section{CORRESPONDENCIA:}

José Ramón González-Escalada

Unidad del Dolor

Hospital Universitario Ramón y Cajal

Ctra. de Colmenar Viejo, km. 9.100

28034 Madrid

e-mail: jr.escalada@telefonica.net

\section{BIBLIOGRAFÍA}

1. Elliott AM, Smith BH, Penny KI, Smith WC, Chambers WA. The epidemiology of chronic pain in the community. Lancet 1999;354:1248-52.

2. Reitsma ML, Tranmer JE, Buchanan DM, Vandenkerkhof EG. The prevalence of chronic pain and pain-related interference in the Canadian population from 1994 to 2008. Chronic Dis Inj Can 2011;31:157-64.

3. Zgierska A, Miller M, Rabago D. Patient satisfaction, prescription drug abuse, and potential unintended consequences. JAMA 2012;307:1377-8.

4. González-Escalada JR, Barutell C, Camba A, Contreras D, Muriel C, Rodríguez M. Creencias, actitudes y percepciones de médicos, farmacéuticos y pacientes acerca de la evaluación y el tratamiento del dolor crónico no oncológico. Rev Soc Esp Dolor 2009;16:7-20.

5. http://neurologia.publicacionmedica.com/spip.php?article540 
6. http://www.medicinatv.com/profesional/reportajes/un-tratamiento-del-dolor-mas-justo-y-equilibrado-en-toda-espana-3890

7. Breivik H, Collett B, Ventafridda V, Cohen R, Gallacher D. Survey of chronic pain in Europe: Prevalence, impact on daily life, and treatment. Eur J Pain 2006;10:287-333.

8. Bosch F, Baños JE. Las repercusiones económicas del dolor en España. Med Clin 2000;115:638-42.

9. Deardorff WW, Rubin HS, Scott DW. Comprehensive multidisciplinary treatment of chronic pain: A follow-up study of treated and non-treated groups. Pain 1991;45:135-43.

10. Flor H, Fydrichc T, Turkd DC. Efficacy of multidisciplinary pain treatment centers: A meta-analytic review. Pain 1992;49:221-30.
11. De la Calle JL, Abejón D, Cid J, del Pozo C, Insausti J, López E y panel de expertos de la Sociedad Madrileña del Dolor. Estándares de calidad asistencial y catálogo de procedimientos de las Unidades de dolor crónico. Rev Soc Esp Dolor 2010;17:114-33.

12. http://www.msssi.gob.es/organizacion/sns/planCalidadSNS/docs/EERR/Unidad_de_tratamiento_del_dolor.pdf

13. http://portal.sedolor.es/contenidos/100/adjuntos/gnpg34cc. pdf

14. http://portal.sedolor.es/contenidos/100/adjuntos/mk46hsxt. pdf

15. Rodríguez Franco L, Cano García FJ, Blanco Picabia A. Evaluación de las estrategias de afrontamiento del dolor crónico. Actas Españolas de Psiquiatría 2004;32:82-91. 